\title{
Cumplimiento de las normas legales y transparencia en el proceso para la designación de abanderados, portaestandartes y escoltas.
}

\section{Compliance with legal regulations and transparency in the process for the designation of standard bearers, standard bearers and escorts.}

Luis David Velasco Almachi ${ }^{1}$., Raúl Yungán Yungán ${ }^{2}$., Mauricio Giovanni López Lasinquiza $^{3}$. \& Mireya Elvia Grefa Yumbo ${ }^{4}$

Recibido: 15-02-2019 / Revisado: 16-02-2019 /Aceptado: 05-03-2019/ Publicado: 04-04-2019

\begin{abstract}
DOI: https://doi.org/10.33262/cienciadigital.v9i2.384

The present article This Article covers the process of choosing of standard bearers, standard bearers and escorts in the educational units, the systematization of the contributions of each of the students of a person is manifested they observe that it is currently fulfilled, supported by the instructions for selecting the dignities of the pavilions, with their structure that is paramount in the difficult work of the authorities, following the practices and codes of the old education system. The purpose of the present investigation is to identify compliance with legal regulations as indicated in the instructions for the election of flag bearers, standard bearers and escorts and teachers of the Juan León Mera Educational Unit "La Salle" of the province of Tungurahua Ambato canton, in order to see Development and compliance. The professionals comply. For this purpose, a self-assessment questionnaire was designed that includes 8 closed questions with short answers. This questionnaire was applied to 5 teachers of the institution. From the results, there are 3 competency profiles (high, medium, low) in the documents that have more and less knowledge, which determines the formative needs of students' success in the institution. The results that the investigation shows; within the
\end{abstract}

\footnotetext{
${ }^{1}$ Universidad Técnica de Ambato, Facultad de Ciencias Humanas y de la Educación, Carrera de Educación Básica. Ambato, Ecuador, email lvelasco4836@uta.edu.ec

${ }^{2}$ Universidad Técnica de Ambato, Facultad de Ciencias Humanas y de la Educación, Carrera de Educación Básica. Ambato, Ecuador. email ryungan@uta.edu.ec

${ }^{3}$ Universidad Técnica de Ambato, Facultad de Ciencias Humanas y de la Educación, Carrera de Educación Básica. Ambato, Ecuador. email mg.lopez@uta.edu.ec

${ }^{4}$ Universidad Técnica de Amato, Facultad de Ciencias Humanas y de la Educación, Carrera de Educación Básica. Ambato, Ecuador, email gmireyaelvia@yahoo.es
} 
educational institutions they know and comply with the legal regulations. In addition, in the commission the presence of the rector is mandatory and it is not necessary the presence of any zonal, district or circuit authority for the election of the flaggers, standard bearers and escorts.

Keywords: choice, pedagogical, dignities, systematization, election process.

\section{Resumen}

El presente artículo comprende el proceso de elección de abanderados, portaestandartes y escoltas en las unidades educativas, se manifiesta la sistematización de los aportes de cada uno de los estudiantes de una persona observan que se cumple en la actualidad apoyado en el instructivo de selección de las dignidades de los pabellones, con su estructura que es primordial en la difícil labor de las autoridades siguiendo las prácticas y los códigos del viejo sistema de educación. La finalidad de la presente investigación es identificar el cumplimiento de la normativa legal como indica el instructivo de la elección de abanderados, portaestandartes y escoltas y docentes de la Unidad Educativa Juan León Mera "La Salle" de la provincia de Tungurahua cantón Ambato, a fin de ver el desarrollo y cumplimiento. Los profesionales lo cumplen. Con este propósito se diseñó un cuestionario de autoevaluación que incluye 8 preguntas cerradas con respuestas cortas. Este cuestionario se aplicó a 5 docentes de la institución. A partir de los resultados se encuentran 3 perfiles competenciales (alto, medio, bajo) en los documentos que tienen mayor y menor conocimiento, que determina las necesidades formativas de éxito de los estudiantes en la institución. Los resultados que muestra la investigación; dentro de las instituciones educativas conocen y dan cumplimiento a la normativa legal. Además, en la comisión la presencia del rector es obligatoria y no es necesaria la presencia de alguna autoridad zonal, distrital o circuito para la elección de los abanderas, portaestandartes y escoltas.

Palabras clave: elección, pedagógica, dignidades, sistematización, proceso de elección.

\section{Introducción.}

A nivel educativo es imprescindible reconocer los méritos realizados por los educandos y verificar si se cumplen las normativas legales al momento de elegir abanderados, portaestandartes y escoltas en las unidades educativas. Por lo tanto, la presente investigación se realiza con el interés de indagar la transparencia del proceso que realiza la comisión encargada de elegir las dignidades de la institución. El impacto que causó dentro de la comunidad educativa será positivo, se dará a conocer el proceso y que se necesita para ser parte de cuadro de honor de una institución educativa.

La educación en el Ecuador ha sido analizada en un contexto globalizado, permitiendo un enfoque en caminada al buen vivir y una labor pedagógica fundamentada en los principios de la educación superior. Los estudios realizados muestran "lo difícil que puede ser 
conseguir una educación orienta al buen vivir mientras existan prácticas y códigos del viejo sistema" (Molina, 2013). La investigación sustenta "la afirmación que la educación orientada a vivir en sociedad", detallado en el Plan Nacional de Desarrollo "Toda una Vida" (Senplades, 2017).

La felicidad no se alcanza por los "bienes materiales sino por el bien ser, a través de este lograr el bien hacer que posibilita el bien tener, para culminar con el buen vivir". Este concepto muestra que el interés colectivo prima sobre el interés individual Molina (2013).

El aporte fundamental de Molina radica en el análisis de la situación de la sociedad, la educación aun conversar código y practicas antiguas que se intenta superar; uno es la elección del abanderado y sus escoltas de la institución educativa como lo establece los artículos 175,176 y 178 del Reglamento de la Ley Orgánica de Educación Intercultural. Por otra parte, la investigación muestra que no se puede lograr una educación orientada al buen vivir, con la elección de los abanderados institucionales se genera una disputa entre estudiantes y los resultados finales será ver ganadores y perdedores. El colectivo Freinet busca en el abanderado su autoformación, el que parte de dos ejes: formar un sujeto autócrata y autorregulado.

La investigación no profundiza en cómo debe ser el perfil del abanderado, el puntaje que debe alcanzar, como es elegido y quienes participan en la elección del abanderado y los escoltas de una institución educativa (Molina Morán, 2013).

Los capítulos de la ley orgánica de educación intercultural han sido analizados de forma global, los estudios realizados muestran grandes cambios en la educación. Según datos oficiales del INEC los índices de analfabetismo se han reducido asi lo afirma Chiriboga Rodríguez, Rocio, \& Gonzabay Cerna (2018). La investigación que la educación es el medio esencial para lograr, transmitir y desarrollar nuestra cultura y además generar conocimiento.

El aporte fundamental de Chiriboga Rodríguez, Rocio, \& Gonzabay Cerna (2018) radica en el análisis del título V del capítulo VII de la Ley Orgánica de Educación Intercultural en donde las instituciones educativas de manera obligatoria deben elegir el cuadro de honor, que estará conformado de: abanderado, portaestandarte y escoltas. Esta distinción será a los nueve estudiantes que hayan obtenido las calificaciones más altas en su vida estudiantil. La ley debe ser expuesta tanto a docentes y estudiantes quienes son responsables de generar una educación de calidad y calidez. Además, las leyes que existe alrededor del mundo buscan el mismo fin mejorar la educación mediante reformas.

La participación es analizada dentro de las instituciones educativas en donde los estudiantes desarrollan un proyecto en busca de crear democracia. (Bambozzi \& Vadori, 2011). La investigación sustenta que la educación que enfocarse a la participación popular de los estudiantes generando una democracia liberal y democracia deliberativa.

El aporte fundamental de Bambozzi \& Vadori (2011) radica en que debe la educación 
dentro de las instituciones educativas debe generar democracia, los estudiantes deben ser entes activos de participación, por otro lado, también al no construir sujetos democráticos es decir no dar los espacios necesarios se promueve un nuevo analfabetismo existirán sujetos marginados y excluidos.

Si bien la investigación aporta a construir una educación en donde se forme sujetos democráticos, no profundiza la participación de los estudiantes en la elección de las dignidades de la institución.

"La representación del poder ha sido analizado en un contexto globalizado ya que desde la antigüedad existía el escolta o guardia de algún rey, general o gobernador" (Reguerra, 2017), p. 53. La investigación sustenta las características principales de un escolta, guardia o militar, eran o son hombres armados dispuestos a cumplir órdenes por sus jefes en un alto rango.

El aporte fundamental de Reguerra (2017) es mostrar el perfil que debe tener un escolta, para ser elegido debía mostrar confianza y lealtad. La confianza es la seguridad del conocimiento que se tiene del otro y la lealtad estar siempre dispuesto a recibir órdenes, además una traición se pagaba con el destierro o la muerte. Por otra parte, demuestran lo duro de cumplir con el cargo de escolta en tiempos pasados, pero no profundiza en las funciones que tienen en la actualidad al ser una dignidad que escoltara al abanderado de una institución educativa.

Los rituales escolares han sido analizados en las instituciones escolares de Argentina los alumnos se aburren y para los docentes se muestra como una carga realizar este tipo eventos culturales que son desarrollados en las escuelas y colegios. Los rituales son presentaciones culturales propias de Argentina. (Guillén, 2008) Este tipo de rituales culturales son importantes ya que sirve para mantener la historia ideológica orinal de nuestros pueblos.

El aporte fundamente de Guillén (2008) es el desarrollo integral de los educandos mediante diversas aproximaciones disciplinarias; como la sociología y la antropología.

Por otra capta la atención, motiva la transmisión de conocimientos ancestrales mismo que son de vital importancia para conocer la historia de nuestro país. No profundiza en la metodología y estrategias para transmitir este conocimiento además no existen temas sobre dignidades educativas.

A través de las observaciones en la vida escolar se considera que la organización general que existe en las instituciones, existe características centrales y dinámicas de funcionamiento institucional y áulico donde la contextualización de las asignaturas tiene un gran impacto en los estudiantes en la construcción de los conocimientos y considerando en las evaluaciones confiables, objetivas, oportunas, pertinentes e imparciales a la prueba ser estudiante proceso que coordina el INEVAL en los saberes tal concordancia de dicho seguimiento a mi concepción es de suma importancia 
promoviendo la calidad de educación. Según el ministerio de educación establece a los planteles educativos como organizadores, desarrolladores del proceso de gestión para mejorar la calidad en los aprendizajes en los estudiantes. Por otro lado, los estándares que ayuda a los docentes, autoridades, estudiantes en si a toda la comunidad educativa desarrollen y contribuyan al óptimo funcionamiento de las instituciones educativas. Gracias a esto y de acuerdo a nuestra investigación cabe recalcar que todo este proceso sistemático a cumplir conllevara a ser parte de la proclamación de abanderados, portaestandartes y escoltas de los pabellones en las unidades educativas fiscales, fiscomisionales, municipales y particulares en el proceso de elección se lo realizara en el transcurso respectivo de elecciones con las debidas apelaciones en caso de ser necesario. Disposiciones que se rigen en las regiones costa, sierra según el decreto 366 del 27 de junio del 2014, suscrito por la ex presidencia de Rafael Correa. (Borja, Manobanda, \& López, 2017, p. 19)

Para Borja (2017) "El objetivo es unificar la celebración de este acto cívico el 26 de septiembre de cada año, con toda la comunidad educativa y sea participe de esta ceremonia tan importante para los estudiantes" El sistema educativo considera que la educación debe centrarse en el ser humano y garantizar su desarrollo en un marco de respeto a los derechos humanos, al medio ambiente y a la democracia, la misma que debe ser participativa, obligatoria intercultural, democrática, incluyente y diversa. Siendo de calidad y calidez además de impulsarla equidad de género, la justicia y la solidaridad, estimular el sentido crítico para su excelente formación como persona y como profesional en condición indispensable para el buen vivir. (Borja, 2017)

El posicionamiento de abanderados y escoltas en centros educativos ha sido analizado en el contexto de la educación universal, permitiendo un enfoque de derechos que potencialice las destrezas de los mejores estudiantes de la nación y el mundo. Los estudios realizados mostraron que en determinados países la xenofobia y el racismo influyen en el posicionamiento de estas dignidades. (Cerutti \& Viard, 1998) La investigación sustenta la afirmación que al designar un abanderado o escolta "se está reconociendo el mejor esfuerzo aplicado en el estudio, que siempre es una contribución al futuro engrandecimiento de la Nación, ya sea que éste lo realice un ciudadano o un extranjero residente en nuestro territorio" (Bariazarra, 1998)

El aporte fundamental de Cerutti \& Viard (1998) radica en el análisis del accionar de las autoridades educativas argentinas al momento de elegir a los abanderados y escoltas estudiantiles en comparación con otros países americanos, proceso que evidencia el patrioterismo existente en el contexto educativo, lo cual es considerado como un atropello a los derechos humanos.

Desde otro punto de vista, la investigación aporta en la construcción de una reglamentación para designar abanderados y escoltas, fundamentada en la Constitución y en los Derechos Humanos, no profundiza en cómo reducir o erradicar la xenofobia y la discriminación en los establecimientos educativos ni mucho menos como garantizar una educación libre de perjuicios. 
La excelencia académica ha sido analizada en el contexto de la inclusión social, permitiendo un enfoque de equidad que deben ser adoptados por la sociedad moderna, los estudios realizados mostraron que los estudiantes que forman parte del Grupo de Alto Rendimiento (GAR) pertenecían a los estratos más bajos siendo excluidos de la educación superior en un sinnúmero de ocasiones. (Mena, Celorio \& Benalcazar, 2003). La investigación sustenta la afirmación que existe una contrariedad entre el discurso propuesto por las autoridades educativas nacionales y la significatividad social del grupo de alto rendimiento (GAR).

El aporte fundamental de Mena, Celorio y Benalcazar (2003) es que el Examen Nacional para la Educación Superior (ENES) no puede predecir el futuro de un estudiante, ya que el ser el mejor puntuado no garantiza que pueda aprobar todas las materias sin ningún inconveniente dentro del ámbito universitario.

Po otra parte si bien la investigación aporta a la inclusión estudiantil en la educación superior sin importar el estrato social, no profundiza en explicar porque se lleva a cabo un examen de razonamiento más no de conocimientos para la admisión a la universidad.

El rendimiento académico ha sido analizado en el contexto escolar permitiendo un enfoque basado en la valoración de los esfuerzos estudiantiles antes que las habilidades, los estudios realizados mostraron que los estudiantes son reconocidos en el salón de clases por su esfuerzo. (Navarro, 2003). La investigación sustenta la afirmación que el uso de estrategias de enseñanza permite conocer el rendimiento escolar estudiantil.

El aporte fundamental de Navarro (2003) radica en la comparación entre el esfuerzo y la habilidad estudiantil, manifestando que el esfuerzo no garantiza el éxito del estudiante ni a corto ni a largo plazo, lo que se vincula claramente con los tipos de inteligencias propuestos por Howard Gardner.

Sin embargo, Jiménez (2000) refiere que "se puede tener una buena capacidad intelectual y una buena aptitud y sin embargo no estar obteniendo un rendimiento adecuado", ante la disyuntiva y con la perspectiva de que el rendimiento académico es un fenómeno multifactorial es como iniciamos su abordaje.

Al respecto, Bandura (1993), en su teoría cognoscitiva social, refiere que la motivación se considera como el producto de dos fuerzas principales, la expectativa del individuo de alcanzar una meta y el valor de esa meta para él mismo. En otras palabras, los aspectos importantes para la persona son, ¿si me esfuerzo puedo tener éxito? y ¿si tengo éxito, el resultado será valioso o recompensante?, la motivación es producto de estas dos fuerzas, puesto que, si cualquier factor tiene valor cero, no hay motivación para trabajar hacia el objetivo.

Por otra parte, si bien la investigación aporta al reconocimiento académico basado en esfuerzos, no profundiza en estrategias de enseñanza globalizadas que se adapten a los intereses y necesidades del estudiantado en general. Es en sí, el compromiso motivacional 
innato que tiene el ser humano por la superación personal.

Elección según (Lynch, 2013) "ésta se propone como la condición para que podamos asignar o atribuir responsabilidad a una conducta. El sujeto moral se convierte en un ente responsable". Para (Kateb, 2011) una dignidad consiste en "la esfera de los valores morales fija su atención en las acciones ya realizadas e implica el reconocimiento del estatus de igualdad, pues los individuos somos igualmente únicos; nadie puede ser simplemente reemplazado por otro". "Ser abanderado o escolta de un plantel educativo es la más alta distinción que puede obtener un estudiante de colegio. Significa poseer numerosos valores como dedicación, disciplina, perseverancia y responsabilidad" (Expreso.ec, 2014). Abanderado es la persona encargada de llevar la bandera o el estandarte en procesiones u otros actos. "Reconocer el mérito escolar del estudiante que haya obtenido el mejor promedio”. (La Nación, 2010). Según la Real Academia de la Lengua Española (RAE) estandarte es "Insignia que usan los cuerpos montados, consistente en un pedazo de tela cuadrado pendiente de una asta, en el cual se bordan o sobreponen el escudo nacional y las armas del cuerpo a que pertenece". "Los escoltas protegen a las personas frente a peligros o a personas que les aborden sin permiso" según John F.

Si consideramos la definición de Jiménez (2000) la cual postula que el rendimiento académico escolar es un "nivel de conocimientos demostrado en un área o materia comparado con la norma de edad y nivel académico"

La motivación escolar es un proceso general por el cual se inicia y dirige una conducta hacia el logro de una meta "Este proceso involucra variables tanto cognitivas como afectivas: cognitivas, en cuanto a habilidades de pensamiento y conductas instrumentales para alcanzar las metas propuestas; afectivas, en tanto comprende elementos como la autoevaluación, auto concepto, etc." (Alcalay y Antonijevic, 1987: 29-32). "Se ha encontrado que los individuos con más altas calificaciones poseen un locus de control interno" (Almaguer, 1998).

La UNESCO United Nations Educational, Scientific and Cultural Organization (Organización de las Naciones Unidas para la Educación, la Ciencia y la Cultura) en su artículo 5 manifiesta que los derechos culturales son parte integrante de los derechos humanos, que son universales, indisociables e interdependientes.

"El desarrollo de una diversidad creativa exige la plena realización de los derechos culturales, tal como los definen el Artículo 27 de la Declaración Universal de Derechos Humanos y los Artículos 13 y 15 del Pacto Internacional de Derechos Económicos, Sociales y Culturales." (UNESCO.ORG, 2001) Por lo tanto, toda persona tiene derecho a una educación y una formación de calidad que respeten plenamente su identidad cultural, dentro de los límites que impone el respeto de los derechos humanos y de las libertades fundamentales.

La Constitución de la República del Ecuador garantiza la educación de todos ciudadanos, 
en su artículo 26, que manifiesta que:

La educación es un derecho de las personas a lo largo de su vida y un deber ineludible e inexcusable del Estado. Constituye un área prioritaria de la política pública y de la inversión estatal, garantía de la igualdad e inclusión social y condición indispensable para el buen vivir. Las personas, las familias y la sociedad tienen el derecho y la responsabilidad de participar en el proceso educativo. (Asamblea Constituyente, 2008)

La educación también se fundamenta en los artículos 27 y 28 de la Constitución que manifiestan lo siguiente:

Art. 27.- La educación se centrará en el ser humano y garantizará su desarrollo holístico, en el marco del respeto a los derechos humanos, al medio ambiente sustentable y a la democracia; será participativa, obligatoria, intercultural, democrática, incluyente y diversa, de calidad y calidez; impulsará la equidad de género, la justicia, la solidaridad y la paz; estimulará el sentido crítico, el arte y la cultura física, la iniciativa.

Art. 28.- La educación responderá al interés público y no estará al servicio de intereses individuales y corporativos. Se garantizará el acceso universal, permanencia, movilidad y egreso sin discriminación alguna y la obligatoriedad en el nivel inicial, básico y bachillerato o su equivalente. (Asamblea Constituyente, 2008)

Según el Registro oficial de la Ley Orgánica de Educación Intercultural expresa: En el Art 2 de los principios en las actividades educativas, desarrollan fundamentos filosóficos, conceptuales y constitucionales que sustentan, definen y rigen las decisiones en todas las actividades que vaya a realizar en las instituciones educativas. (Ministerio de Educación, Actualizada 2015) Dicho esto, en el literal i, con respecto a la educación en valores, "se basa en la práctica de valores que fomentan libertad, democracia, respeto a los derechos responsabilidad, solidaridad, tolerancia diversidad de género, etnia, social, identidad de género, creencias, equidad, justicia, ya la erradicación de la discriminación” (Ministerio de Educación, Actualizada 2015)

Con respecto a la corresponsabilidad en el literal p, donde la educación es responsable en la formación e instrucción en niños, niñas y adolescentes una educación digna, donde está vinculada toda la comunidad educativa. (Ministerio de Educación, Actualizada 2015)

Y gracias a la motivación que manifiesta el literal q, de la presente ley esta promueve el esfuerzo individual en su aprendizaje, obteniendo un mejor desarrollo personal donde dará cumplimiento el estudiante a sus derechos y obligaciones que son factores esenciales a la calidad de educación. (Ministerio de Educación, Actualizada 2015)

"En el Art 8 de obligaciones manifiesta que los estudiantes tienen obligaciones al dar cumplimiento de tareas, procurando la excelencia educativa mostrando su integralidad y honestidad académica dicho en el literal c de la presente ley" (Ministerio de Educación, Actualizada 2015) 
En el Art 13 del reglamento a la Ley Orgánica de Educación Intercultural manifiesta que las madres, padres y/o representantes legales tienen la obligación como dicta el literal h, de reconocer el mérito y excelencia académica de los docentes sin que implique erogación económica. (Ministerio de Educación, Actualizado 2015)

Según el Art. 175 del Reglamento a la Ley Orgánica de Educación Intercultural (Ministerio de Educación, Actualizado 2015) todas las instituciones educativas del país deberán elegir entre los estudiantes del tercer año de bachillerato "al abanderado del Pabellón Nacional con sus dos escoltas. Además, elegirán al portaestandarte de la Ciudad (o del Cantón) y al portaestandarte del Plantel, con dos escoltas en cada caso"

Según el Art. 176 del Reglamento a la Ley Orgánica de Educación Intercultural (Ministerio de Educación, Actualizado 2015) y en el Instructivo para designación de abanderados, portaestandartes y escoltas, estas distinciones se denominara 9 estudiantes de tercer curso, que hayan logrado el más alto puntaje a los largo de su vida estudiantil. Será el resultado obtenido al promediar las notas finales de aprovechamiento de la Educación General Básica y Bachillerato. De acuerdo a los puntajes se eligen a las dignidades en el siguiente orden:

- Primer puesto: Abanderado del Pabellón Nacional

- Segundo puesto: Portaestandarte de la Ciudad (o del Cantón)

- Tercer puesto: Portaestandarte del Plantel

- Cuarto y quinto puestos: Escoltas del Pabellón Nacional

- Sexto y séptimo puestos: Escoltas del Estandarte de la Ciudad

- Octavo y noveno puestos: Escoltas del Estandarte del Plantel (Acuerdo ministerial No 180-11, 2012)

"Para evitar en lo posible los empates, el promedio final de aprovechamiento se calculará en décimas, centésimas y milésimas. En ningún caso esta cifra se promediará con las notas de conducta o disciplina" (Ministerio de Educación, Actualizado 2015)

De acuerdo a los parámetros de calificaciones se designa a los estudiantes en el siguiente orden, abanderado, portaestandarte y escoltas.

Tabla 1

\begin{tabular}{|c|c|}
\hline Escala cualitativa & Escala cuantitativa \\
\hline Domina los aprendizajes requeridos & $9,00-10,00$ \\
\hline Alcanza los aprendizajes requeridos & $7,00-8,99$ \\
\hline Está próximo a alcanzar los aprendizajes requeridos & $4,01-6,99$ \\
\hline No alcanza los aprendizajes requeridos & $\leq \mathbf{4}$ \\
\hline
\end{tabular}

Fuente: Reglamento a la ley Orgánica de Educación Intercultural 
Elaborado por: Autores

Y por si fuera los empates de acuerdo al Art 177 del presente reglamento (Ministerio de Educación, Actualizado 2015), en un empate los promedios globales para desempatar se considerarán como adicionales participaciones de otras actividades sean estos científicos, culturales, artísticas, deportivas siempre y cuando se tenga respaldo en archivos. Optimizando el respaldo del código de convivencia del establecimiento educativo, siendo esta debidamente comprobada en la dirección distrital.

Art. 179.- Se conformará una comisión para la elección de abanderados, portaestandartes y escoltas, integrada por cinco miembros:

a. El Rector del plantel, quien la presidirá;

b. Dos delegados del Consejo Ejecutivo del establecimiento;

c. Un representante de los Padres y Madres de Familia; y,

d. El Presidente del Consejo Estudiantil. (Ministerio de Educación, Actualizado 2015)

Según el artículo 179 esta comisión debe tener un secretario, este cargo ocupa el secretario del plantel; en caso de no existir se elegirá un secretario ad hoc de preferencia un docente. Su función es dar fe de lo ocurrido en la reunión solo tendrá voz y no voto. Además, participaran otras personas que actuaran como veedores con voz, pero sin voto, cuya participación debe constar en el Código de Convivencia de la Institución Educativa. Reglamento a la Ley Orgánica de Educación Intercultural (Ministerio de Educación, Actualizado 2015)

Art. 180.- Para hacerse acreedor a las distinciones de Abanderado. Portaestandarte o Escolta, un estudiante deberá cumplir con los siguientes requisitos:

1. Estar legalmente matriculado en el tercer año del Bachillerato del establecimiento educativo y asistir de forma regular a clases;

2. Haber entregado en la secretaría del plantel copias legalizadas de los pases de un año los registros con las calificaciones obtenidas desde segundo año de EGB hasta segundo año de Bachillerato, solamente en el caso de estudiantes que cursaron esos años en otros planteles. Reglamento a la Ley Orgánica de Educación Intercultural (Ministerio de Educación, Actualizado 2015)

Según el Reglamento a la Ley Orgánica de Educación Intercultural (Ministerio de Educación, Actualizado 2015) Art.181.- La elección de los abanderados, portaestandartes y escoltas se realizará en un plazo de 10 días laborales desde el inicio del año lectivo. El rector entregará un comunicado al estudiante y representante legal indicando la nominación. Además, se publicará los resultados dentro de la institución por 10 días. 
Según el Art. 182.- en el caso de estar inconforme ante la decían tomada los estudiantes con su representante legal debe impugnar la decisión tomada en un plazo de 5 días. Primero, deberán recurrir a la comisión con su impugnación debidamente sustentada, y la comisión en máximo 5 días laborables deberá dar una respuesta. Por último, podrán apelar, dentro de un plazo de cinco días laborales a partir de la fecha de respuesta de la comisión, ante el Director Provincial de Educación quien deberá resolver el caso en un máximo de cinco días laborales mediante una resolución administrativa motivada. Reglamento a la Ley Orgánica de Educación Intercultural (Ministerio de Educación, Actualizado 2015)

La fecha de la proclamación y juramento expuesto por el art 183 manifiesta que la proclamación de las dignidades se llevará a cabo el 26 de septiembre de cada año día de celebración de la bandera nacional evento que se lo realizará ante toda la comunidad educativa. Evento tan importante para el recibimiento de las dignidades acreedoras de su máximo esfuerzo. (Ministerio de Educación, Actualizado 2015)

\section{Metodología.}

La investigación realizada fue de campo, participaron 5 docentes de la Unidad Educativa Juan León Mera "La Salle" de la provincia de Tungurahua cantón Ambato. La técnica y el instrumento aplicado fue creado por nuestro equipo de investigadores, las preguntas del cuestionario están enfocadas a conocer el proceso y la transparencia al momento de elegir a los abanderados, portaestandartes y escoltas de las unidades educativas.

El cuestionario consta de 8 preguntas, 6 de ellas con dos alternativas: Si y No; y 2 preguntas con tres alternativas: Siempre, A veces, Nunca. Esta determina como se realiza el proceso dentro de las instituciones, fue aplicada dentro de la institución a 3 docentes, el coordinador pedagógico y el Rector. La validación de la información obtenida se realizó con el método alfa de Cronbach, dando como resultado una confiabilidad alta 0,981 .

\section{Resultados.}

La Unidad Educativa cumple a cabalidad lo dispuesto en el Reglamento General de la Ley Orgánica de Educación Intercultural, lo que dispone en sus artículos del 175 al 183, en referencia a la normativa para la designación de abanderado, portaestandarte y escoltas, así como, a lo reglado en el instructivo para el efecto, la entidad mencionada cumple a cabalidad. Que, para dar cumplimiento a lo dispuesto en las normativas legales en cumplimiento al Art. 179 del mencionado reglamento, se conforma la comisión de manera oportuna.

Para las designaciones la comisión encargada realiza una revisión previa de documentos probatorios de cada uno de los estudiantes, verificando el fiel cumplimiento de las disposiciones dada por las Autoridades del Ministerio de Educación, así como, de las prescritas en el Reglamento de la LOEI. En la parte operativa la comisión se reúne en las 
fechas establecidas, para examinar las carpetas académicas de cada uno de los estudiantes, como la obtención de los promedios y otros méritos.

Luego de haber cumplido con el estudio del record estudiantil y su análisis pertinente, corroborando y evidenciando la transparencia del proceso, designan en orden de puntajes de mayor a menor a los estudiantes que conformará el Cuadro de Honor para el respectivo periodo académico. Por lo que en uso de sus atribuciones la Comisión para la elección de Abanderado o Abanderada, Portaestandartes y Escoltas, resuelven designar las distinciones en orden de puestos portaestandarte con los nombres respectivos de cada uno de los y las estudiantes.

Los resultados finales elevan en un acta de designación de Abanderado, Portaestandartes y Escoltas generada por el aplicativo informático, deberá ser suscrita por los miembros de la Comisión y entregada oficialmente al Directivo Institucional para su publicación y conocimiento de la comunidad educativa, tal como se describe en el Instructivo de DESIGNACIÓN DE ABANDERADOS, PORTAESTANDARTES Y ESCOLTAS, expedido por el Ministerio de Educación.

En el mismo documento reconocen, felicitan y solicitan entregar por escrito a los estudiantes galardonados sus respectivas designaciones y hacer público en el acto cívico de Proclamación de Abanderados, Portaestandartes, Escoltas y Juramento a la Bandera, que se hará en la respectiva fecha. De la misma manera las autoridades de la Institución educativa manifiestan que se le da la apertura y atención oportuna en caso de que hubiere apelaciones, de acuerdo a los que establece y la misma normativa legal para receptar el recurso de apelación interpuesto por el representante legal del estudiante dentro de un período no mayor a cinco (5) días laborables.

Por último, la proclamación de Juramento a la Bandera en la Institución cumple a cabalidad con la participación de todos los actores educativos, de conformidad a lo prescrito en el artículo 183 sustituido mediante el Decreto Ejecutivo $\mathrm{N}^{\circ} 366$ de 27 de junio de 2014, publicado en el Segundo Suplemento del Registro Oficial $N^{\circ} 286$ de 10 de julio de 2014.

Se puede evidenciar que la Unidad Educativa Juan León Mera "La Salle", es una Institución que cumple con las normativas legales del Ecuador y con la visión, misión y sobre todo con los valores institucionales.

En la investigación de campo se procedió a aplicar las encuestas las mismas que se fueron analizadas e interpretadas ¿Conoce la normativa legal para designar abanderados, portaestandartes y escoltas dentro de la Institución?, datos presentados en la tabla $\mathrm{N}^{\circ} 2$, en 5 encuestados el $80 \%$ de los docentes manifiestan que conocen sobre la normativa legal, además que es aplicado con rigor los artículos del Reglamento a la Ley Orgánica de Educación Intercultural con la finalidad de no perjudicar a los estudiantes y cumplir lo legal. 
Tabla 2

\begin{tabular}{|c|c|c|c|c|}
\hline \multicolumn{5}{|c|}{ Conocen la Normativa Legal } \\
\hline Alternativas & Frecuencia & Porcentaje & Porcentaje válido & Porcentaje acumulado \\
\hline Si & 4 & 80 & 80 & 80 \\
\hline No & 1 & 20 & 20 & 100 \\
\hline Total & $\mathbf{5}$ & $\mathbf{1 0 0}$ & $\mathbf{1 0 0}$ & \\
\hline
\end{tabular}

Fuente: Encuesta aplicada a docentes

Elaborado por: Autores

Un 60\% manifiesta que es no necesario la presencia de veedores dentro de la comisión de elección de abanderados. Por otro lado, un $100 \%$ manifestó que no es necesaria dentro la comisión la presencia de alguna autoridad zonal, distrital o circuital. Por otra parte, un $100 \%$ manifestó que es importante la presencia del Rector, Datos presentados en la tabla $\mathrm{N}^{\circ} 3$, argumentan que es el encargado de dirigir la comisión de elección de abanderados, portaestandartes y escoltas.

Tabla 3

\begin{tabular}{|c|c|c|c|c|c|}
\hline \multicolumn{6}{|c|}{ Autoridad Zonal, distrital o circuital o presencia del Rector } \\
\hline \multicolumn{2}{|c|}{ Alternativas } & Frecuencia & Porcentaje & $\begin{array}{c}\text { Porcentaje } \\
\text { válido }\end{array}$ & $\begin{array}{c}\text { Porcentaje } \\
\text { acumulado }\end{array}$ \\
\hline $\begin{array}{c}\text { Presencia } \\
\text { de alguna } \\
\text { Autoridad } \\
\text { Zonal. }\end{array}$ & No & 5 & 100 & 100 & 100 \\
\hline $\begin{array}{c}\text { Presencia } \\
\text { del Rector }\end{array}$ & Si & 5 & 100 & 100 & 100 \\
\hline
\end{tabular}

Fuente: Encuesta aplicada a docentes

Elaborado por: Autores

Un 100\% de encuestados manifestaron que al momento de elegir los abanderados a veces existen empates, los mismos que son resueltos mediante la calificación de méritos, distinciones o logros académicos de los estudiantes tales (deportes, música, exposiciones, actos religiosos, programas culturales). También se resuelven tomando en cuenta las décimas, centésimas y milésimas. Esta resolución está a cargo del tribunal o la comisión de elección de abanderados, portaestandartes y escoltas.

Las autoridades de la institución manifestaron que, hasta el momento, ningún estudiante ha apelado la decisión tomada por la comisión, al contrario, tanto los estudiantes, padres de familia y representantes legales han demostrado su satisfacción con felicidad y orgullo por el logro alcanzado a lo largo de la vida estudiantil de sus hijos y/o representados. Esto demuestra que la institución actúa con transparencia guiándose y rigiéndose a los artículos 
del Reglamento a la Ley Orgánica de Educación Intercultural y el Instructivo para la designación de abanderados, portaestandartes y escoltas.

En este mismo sentido el $60 \%$ de encuestados, manifestaron que, en el caso de existir alguna irregularidad por parte de la comisión en la designación de las dignidades, existen sanciones administrativas, tales como la separación de la comisión y llamado de atención por escrito, con la finalidad de respetar el derecho de los estudiantes.

\section{Conclusiones.}

- En conclusión, el trabajo que realiza la comisión de elección de abanderados, portaestandartes y escoltas al elegir dichas dignidades lo realiza con transparencia y eficacia con la finalidad de no perjudicar a los estudiantes, por el contrario, busca reconocer el mérito alcanzado en la trayectoria estudiantil.

- Se evidencia que la Unidad Educativa Juan León Mera "La Salle", es una Institución que cumple con las normativas legales del Ecuador y con la visión, misión y sobre todo con los valores institucionales, por lo que garantiza los derechos de niños y niñas, sus maestros, maestras, autoridades y padres de familia están conscientes de estimular el espíritu de superación del estudiante, reconociendo el esfuerzo y dedicación en las actividades académicas, sociales, culturales, deportivas y espirituales.

- Los procesos de elección de abanderados, portaestandartes y escoltas en las unidades educativas se realizan con la veeduría de autoridades educativas gubernamentales, sin embargo, el personal docente y las autoridades educativas institucionales manifiestan que no es necesario su presencia, puesto que dichos procesos se realizan con transparencia y en base a su código de ética.

- Dentro de la comisión para la elección de abanderados, portaestandartes y escoltas, es la señora Rectora, quien preside el tribunal, junto a los delegados del consejo ejecutivo, el representante de los padres de familia y el representante del consejo estudiantil. No es necesario la presencia de veedores y en el caso de existir tendrán voz y no voto al igual que la secretaria que participa dentro de la comisión. Se concluye que la institución asume con responsabilidad la participación de los actores educativos.

- Al momento de presentarse alguna irregularidad al momento de elegir las dignidades de una institución educativa si existe una sanción administrativa esta puede ser un llamado de atención o la destitución dentro de la comisión, así se respeta el derecho de los estudiantes acreedores a esta designación y se realiza el proceso con transparencia en base a la ley.

- Por lo tanto, la educación es derecho de todos los seres humanos, la cual existirá autoridades competentes para llevar a cabo con dignidad y transparencia el realce 
de los estudiantes mejores puntuados para dicho proceso de designación de abanderados, portaestandartes y escoltas en las Unidades Educativas Escolares de la República del Ecuador.

\section{Referencias Bibliográficas}

Acuerdo ministerial No 180-11. (2012). NORMATIVA PARA LA DESIGNACIÓN DE ABANDERADOS, PORTAESTANDARTES Y ESCOLTAS. Quito.

Asamblea Constituyente. (2008). Constitución de la República del Ecuador. Alfaro.

Bambozzi, E., \& Vadori, G. (2011). Educación y democracia: La construcion Política en las instituciones educativas en perspectivas pedagógicas. Scielo, 1-11. Obtenido de http://www.memoria.fahce.unlp.edu.ar/trab_eventos/ev.944/ev.944.pdf

Borja, R. V. (2017). El Proceso Educativo Fiscal Y Su Influencia En Los Resultados De Las Pruebas Ser Estudiante, Del Séptimo Año De Educación General Básica, En El Cantón Guaranda, Ecuador. European Scientific Journal, 10.

Cerutti, Á., \& Viard, G. (1998). De bandera, abanderados y extranjeros en las escuelas neuquinas. Consideraciones sobre el tratamiento del problema en el diario "La Mañana del Sur”, diciembre de 1998. Scripta Ethnologica, 31-38.

Chiriboga Rodríguez, J. C., Rocio, M. F., \& Gonzabay Cerna, J. B. (2018). Sinopsis de los titulos V-VI de la Ley orgánica de educación Intercultural ecuatoriana. Mapa, 307-318.

Obtenido

de http://www.revistamapa.com/index.php/es/article/view/85/68

Expreso.ec. (20 de mayo de 2014). Abanderados cuentan lo que significa este honor. Expreso.

Guillén, I. C. (2008). Los rituales escolares en la escuela pública polimodal Argentina. Scielo, $1-17$. Obtenido de http://www.scielo.org.ar/scielo.php?script=sci_arttext\&pid=S185116942008000100008

Kateb, G. (2011). Dignidad Humana. Scielo.

La Nación. (2010). "Ser abanderado en la Argentina es un logro que vale la pena" . La Nación.

Lynch, E. (2013). LA ELECCIÓN Y LA DECISIÓN. El Nubarrón.

Ministerio de Educación. (Actualizada 2015). Ley Orgánica de Educación Intercultural. Quito.

Ministerio de Educación. (Actualizado 2015). Reglamento a la Ley Orgánica de 
Educación Intercultural. Quito.

Molina Morán, E. (2013). Ecuador: la problemática de la elección del abanderado en una educación orientada al buen vivir. Redalyc.org. Obtenido de http://www.redalyc.org/html/270/27028897004/

Reguerra, A. (2017). Los edecanes de Juan Manuel de Rosas. La confianza en la delegación y representación personal del poder. Scielo, 52-70. Obtenido de www.scielo.org.ar/pdf/trav/v19n1/v19n1a03.pdf

UNESCO.ORG. (2 de Noviembre de 2001). Declaración Universal de la UNESCO sobre la Diversidad Cultural. Obtenido de http://portal.unesco.org/es/ev.phpURL_ID=13179\&URL_DO=DO_TOPIC\&URL_SECTION=201.html

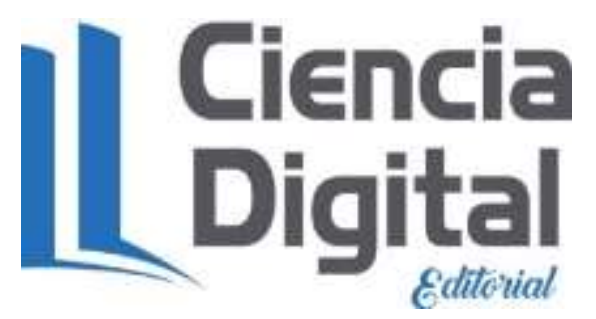




\section{Para citar el artículo indexado.}

Velasco L., Yungán R., López M. \& Grefa M. (2019). Cumplimiento de las normas legales y transparencia en el proceso para la designación de abanderados, portaestandartes y escoltas. Revista electrónica Ciencia Digital 3(2), 181-197. Recuperado desde: http://cienciadigital.org/revistacienciadigital2/index.php/CienciaDigital/article/view/384 $\underline{1832}$

\section{Liencia}

El artículo que se publica es de exclusiva responsabilidad de los autores y no necesariamente reflejan el pensamiento de la Revista Ciencia Digital.

El artículo queda en propiedad de la revista y, por tanto, su publicación parcial y/o total en otro medio tiene que ser autorizado por el director de la Revista Ciencia Digital.
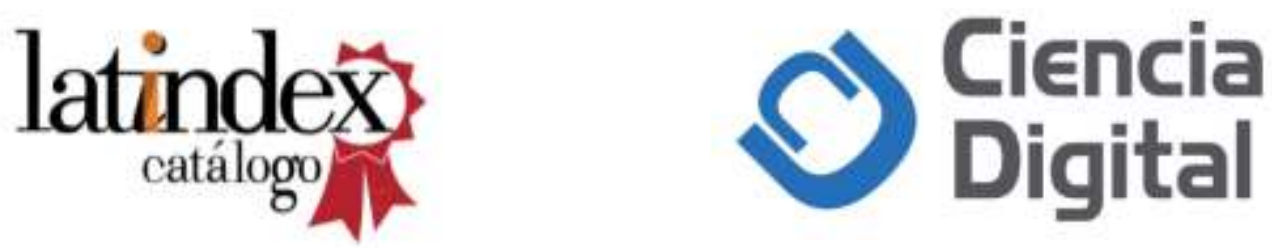